\title{
Ikaeria serusiauxii, a new Caloplaca-like lichen from Macaronesia and mainland Portugal, with a lichen checklist for Porto Santo
}

\author{
Harrie J. M. Sipman ${ }^{1}$ \& André Aptroot ${ }^{2,3}$
}

\section{Article info}

Received: 12 Sept. 2019

Revision received: 22 Oct. 2019

Accepted: 4 Nov. 2019

Published: 2 Jun. 2020

Associate Editor

Nicolas Magain

\begin{abstract}
The new species Ikaeria serusiauxii (Teloschistaceae, lichenized Ascomycetes) is described from the Madeira Archipelago, Canary Islands and continental Portugal. It is a crustose lichen on twigs and branches of trees and shrubs in xerophytic maritime vegetation. Superficially it is similar to Caloplaca cerina and $C$. haematites, from which it differs by the often black apothecium margin, very thick spore septa, black pycnidium ostioles, and the presence of the pigment Cinereorufa-green instead of Sedifolia-grey. ITS sequences suggest Ikaeria aurantiellina (syn. Caloplaca aegatica) as the closest relative. Added is a preliminary lichen checklist for Porto Santo (Madeira Archipelago, Macaronesia).
\end{abstract}

Key words: Taxonomy, lichens, diversity, island biology

\section{Introduction}

The Madeira Archipelago, one of the island groups of Macaronesia, is situated in the Atlantic Ocean some $500 \mathrm{~km}$ off the shore of NW Africa. Politically it belongs to Portugal. Like the Canary Islands, it has a dry warm climate except where higher mountains cause increased precipitation. During a visit to Porto Santo, the second largest island of the Madeira Archipelago, for a lichen mapping project (Sparrius et al. 2017), an unusual Caloplaca-like, epiphytic lichen showed up frequently on shrubs and trees, which somewhat resembled C. cerina or C. haematites. Morphological and macromolecular analyses showed it to be an undescribed species, which is treated below. Further results of this expedition are presented at https://archive.bgbm.org/sipman/Zschackia/ PortoSanto/genuslist.htm, and a preliminary checklist for Porto Santo is presented below (Table 1).

\section{Material and methods}

Specimens were studied with a stereomicroscope and a compound microscope in tap-water mounts. ITS sequences were generated by Alvalab (Spain). The

\footnotetext{
${ }^{1}$ Botanischer Garten \& Botanisches Museum, Freie Universität, Koenigin-Luise-Str. 6-8, D-14195 Berlin, Germany

${ }^{2}$ BL Herbarium, G. v.d. Veenstraat 107, NL-3762 XK Soest, The Netherlands

${ }^{3}$ Laboratório de Botânica / Liquenologia, Instituto de Biociências, Universidade Federal de Mato Grosso do Sul, Avenida Costa e Silva s/n, Bairro Universitário, CEP 79070-900, Campo Grande, Mato Grosso do Sul, Brazil

* Corresponding author e-mail: H.Sipman@bgbm.org
}

sequences were analysed using https://www.ebi.ac.uk/ Tools/msa/muscle/ with standard settings and http://iqtree. cibiv.univie.ac.at/ (Trifinopoulos et al. 2016) with standard settings and sequence type = DNA (accessed 18 June 2019). Branch support values were obtained with ultrafast bootstrap (Hoang et al. 2018) implemented in IQ-TREE (Nguyen et al. 2015).

Vouchers are deposited in B, BR, M, MADJ and herb. van den Boom.

\section{Results and discussion}

For complete documentation of the new species, ITS sequences were generated. These gave a preliminary view of the affinities of the new species. A BLAST search in Genbank in 2017 gave the surprising result that the closest relatives were in the genus Lecidea. A repeated BLAST search in 2019 suggested an affinity with the genus Ikaeria, which was published meanwhile by Kondratyuk et al. (2017). It comprises the single species Ikaeria aurantiellina, based on samples from Tenerife, Canary Islands. The genus was found to belong to the subfamily Teloschistoideae as sister to the genus Yoshimuria, and not to the Caloplacoideae or Xanthorioideae where most crustose 'Caloplaca' species in Europe and the Mediterranean belong.

Following these suggestions, a comparison of the new species with putative relatives was made. ITS sequences, mostly downloaded from Genbank, were aligned with Megalospora, Brigantiaea and Letrouitia as outgroups, with Caloplaca cerina and C. haematites 


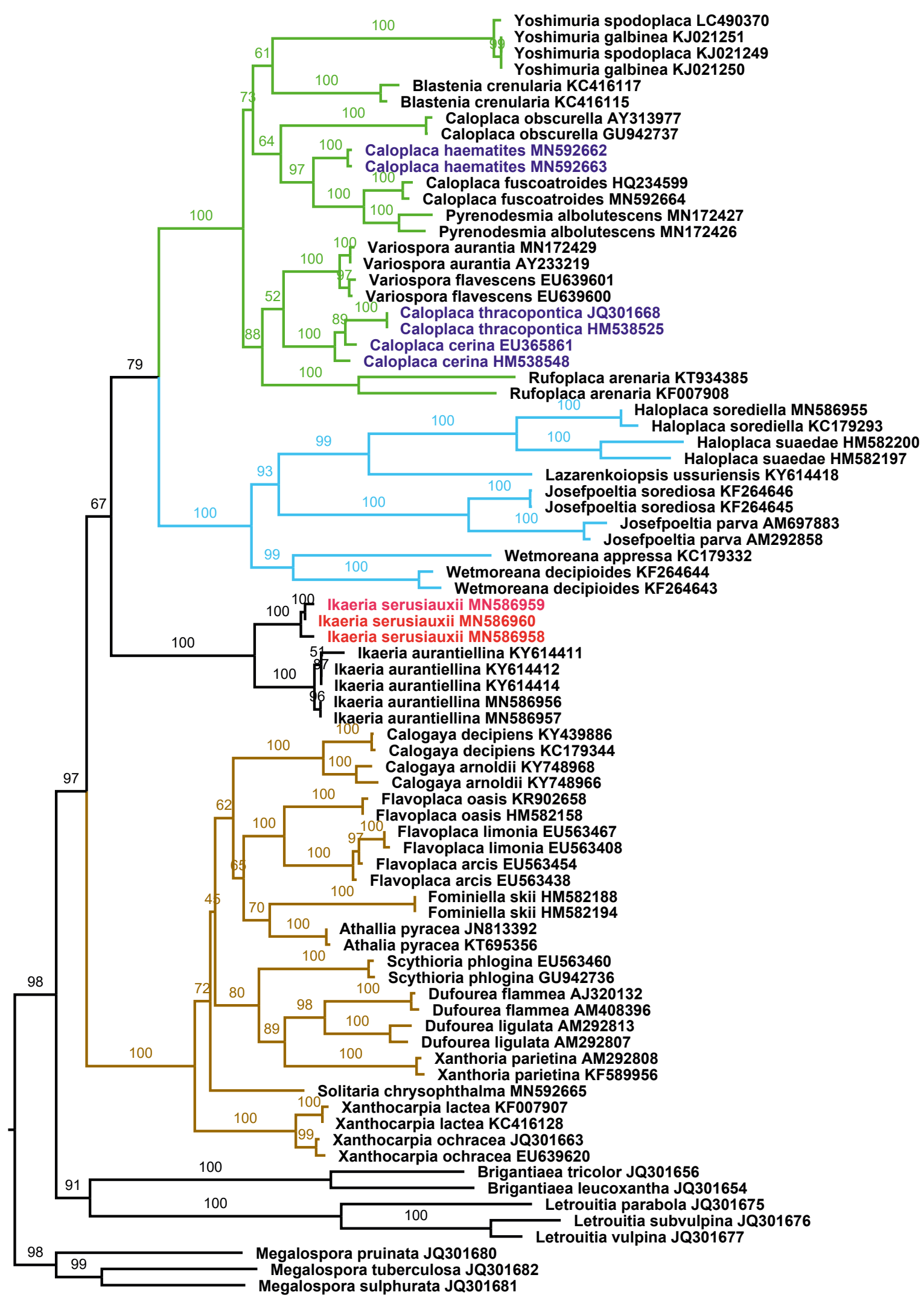

Figure 1. Phylogenetic tree from ITS sequences, with UFBootstrap values, of Ikaeria serusiauxii and selected Teloschistaceae. I. serusiauxii appears not closely related to Caloplaca cerina or C. haematites (blue) and falls outside the most frequent subfamilies in Macaronesia and Europe, i.e. Caloplacoideae (green clade) and Xanthorioideae (brown clade). Its closest relative is I. aurantiellina (black), which is included in Teloschistoideae (blue clade) in multilocus trees. Terminal bootstrap values omitted. 
as potential relatives, with the genus Ikaeria, and with selected representatives of the three main groups of Teloschistaceae, the subfamilies Xanthorioideae, Caloplacoideae and Teloschistoideae (Arup et al. 2013). The resulting tree (Fig. 1) shows that the new species is clearly distinct from $C$. cerina and $C$. haematites, and that it has Ikaeria aurantiellina as the closest relative. Therefore the new species is included in the genus Ikaeria.

From Porto Santo, where the new species was recognized first, few lichen species have been reported so far. Krog \& Østhagen (1980) and Krog (1990) reported Ramalina species, and Haugan (1992) a species of Anzia. These authors discovered remarkable lichen endemism on the island. Short lists of additional species were published by Follmann (1990), Carvalho et al. (2008) and Sparrius et al. (2017). Some recent monographers included material from the island, in particular Timdal (1992) on Toninia. The presented checklist (Table 1) is based mainly on the more easily recognizable lichen species observed during our mapping fieldwork. An attempt was made to study some groups in more detail, but the example of Ikaeria serusiauxii showed that a full evaluation requires more effort than we can invest currently. Therefore we are using the opportunity to publish all data collected so far in a checklist, including information on whether TLC was done, and we release all newly generated ITS sequences, including for groups for which no conclusive taxonomy is settled yet.

Ikaeria serusiauxii Sipman, sp. nov.

(Figs 2-3)

MycoBank MB 833026

Diagnosis: similar to Caloplaca cerina in its anthraquinone-free thallus and apothecia with orange discs and often grey margin, but differing in having black pycnidium ostioles, thick ascospore septa, and the presence of the pigment Cinereorufa-green instead of Sedifolia-grey.

Type: Portugal, Madeira Islands, Porto Santo: E part, lower slopes N of Pico do Facho; $\sim 350 \mathrm{~m} ; 33^{\circ} 05.2^{\prime} \mathrm{N}, 16^{\circ} 19.3^{\prime} \mathrm{W}$; epiphytes on fallen Pinus trees on slope; 2 March 2016; H. Sipman 62971 (B 600200928 - holotype; MADJ - isotype). ITS sequence: MN586960; LSU: MN586916: SSU: MN586910.

Description. Thallus continuous, $\sim 1-3 \mathrm{~cm}$ wide, grey, in shade with a greenish or slightly brownish tinge, not pruinose, smooth and slightly glossy, $\sim 0.05 \mathrm{~mm}$ thick, not sorediate or blastidiate, flat or slightly warty with low warts $0.1-0.2 \mathrm{~mm}$ wide; prothallus black, visible along the thallus margins and on abraded spots; cortex 10-20 $\mu \mathrm{m}$ thick, prosoplectenchymatous, composed of periclinal hyphae; algal layer $\sim 30-50 \mu \mathrm{m}$ thick, discontinuous; medulla absent. Apothecia zeorine, abundant,
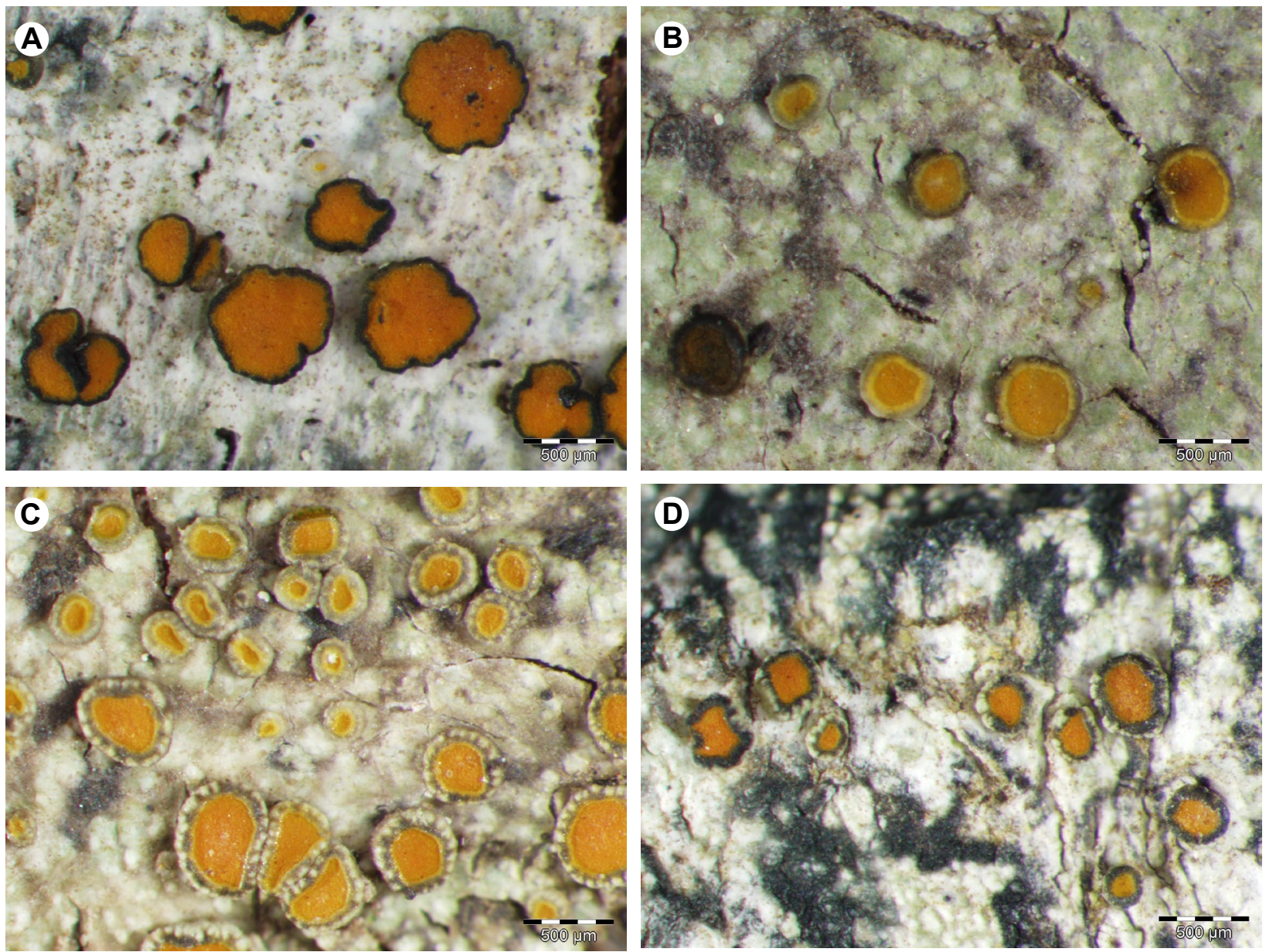

Figure 2. Ikaeria serusiauxii. A - apothecia in full light, with completely black margins (Sipman 62798); B - apothecia in shade, with grey margin (Sipman 62802); C - apothecia in shade, with grey-crenulated margin (Sipman 62957); D - apothecia in full light, with black-crenulated margin (Sipman 62971, holotype). Note black prothallus visible in abraded parts of thallus. Scales: A-D = 500 $\mu \mathrm{m}$. 

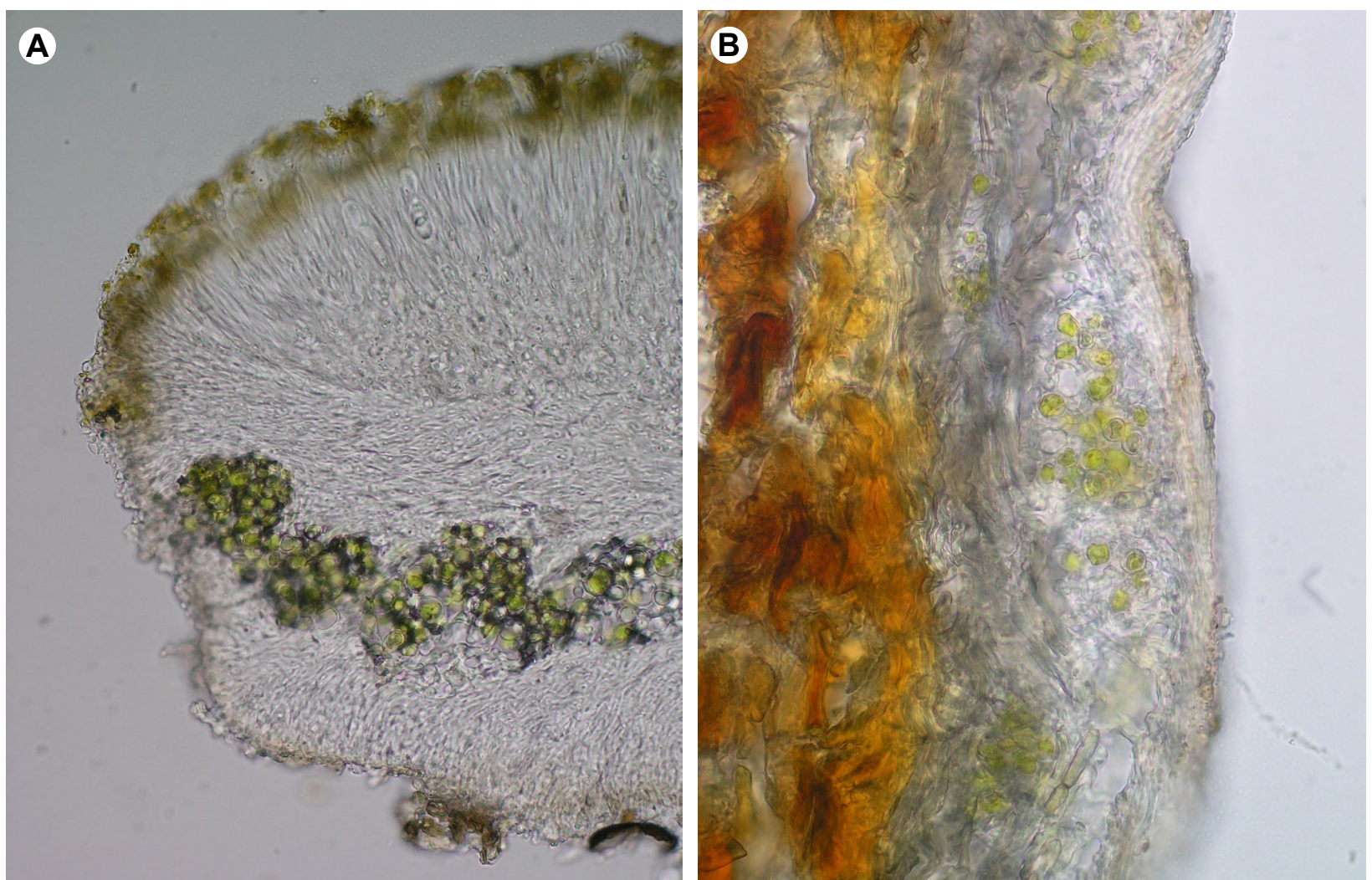

Figure 3. Anatomy of Ikaeria serusiauxii. A - apothecium section showing prosoplectenchymatic proper excipulum and cortex; B - thallus section showing thin, prosoplectenchymatic cortex and absence of medulla. Scale: large algal cells in A and B.

$\sim 0.5-0.8 \mathrm{~mm}$ in diam., when wider mostly subdivided into a few marginate discs forming a convex group, sessile, non-pruinose; disc flat to slightly convex, orange; margin of variable color ranging_from completely black to greenish grey, more commonly intermediate, greenish grey with black spots in marginal crenulations, raised above the disc when young, somewhat reduced in old apothecia; true exciple and hypothecium prosoplectenchymatous, $\sim 10-30 \mu \mathrm{m}$ thick; thalline exciple $\sim 100 \mu \mathrm{m}$ thick; cortical layer $\sim 50 \mu \mathrm{m}$ thick below, thinner laterally, composed of dense, branching, anticlinal hyphae; algal layer $\sim 50 \mu \mathrm{m}$, interrupted, with Trebouxia-like algae $\sim 6-10 \mu \mathrm{m}$ in diam.; epihymenium orange, granular; hymenium 50-60 $\mu \mathrm{m}$ thick, hyaline; paraphyses simple for most of their length, $\sim 2 \mu \mathrm{m}$ wide, apically slightly swollen to $\sim 3 \mu \mathrm{m}$ and dichotomously branched a few times; ascospores polarilocular, ellipsoid, $\sim 12-16 \times$ 6-8 $\mu \mathrm{m}$; septum 8-12 $\mu \mathrm{m}$ wide; ratio of septum width to spore length 0.6-0.75. Pycnidia scattered, rather sparse, immersed with \pm raised black ostiole; conidia bacilliform, $\sim 3.5 \times 0.8 \mu \mathrm{m}$.

Chemistry. Not tested by TLC; the black parts of the apothecia, the pycnidium ostiole and the prothallus contain dark olive-green pigment in the outer locules of the cortex, in $\mathrm{K}$ turning more greenish but persistent (Cinereorufa-green); the epithecium turning violet in $\mathrm{K}$, releasing clouds of fine violet crystals (indet. anthraquinones); thallus and apothecium margin lack anthraquinones $(\mathrm{K}-)$.

Etymology. Named after Emmanuel Sérusiaux, our esteemed companion on expeditions in Papua New
Guinea, who contributed significantly to the exploration of the lichen diversity of Macaronesia.

Distribution and ecology. The species is known from Macaronesia (Madeira Archipelago and Canary Islands) and from mainland Portugal (Algarve, Estremadura). Here it is found on twigs and branches of trees and shrubs in open, rather xerophytic vegetation, e.g. on Euphorbia piscatoria, but also on introduced Cupressus and Pinus. On Porto Santo it is fairly common at 350-400 m a.s.l. From the Madeira Island, so far two records are available, from $500-575 \mathrm{~m}$ a.s.l. The localities in mainland Portugal are close to the seashore.

Notes. Caloplaca cerina is the most likely species to be confused with Ikaeria serusiauxii, as it shares an anthraquinone-free, pale thallus, anthraquinone-free apothecium margins and yellow to orange-colored discs (Šoun et al. 2011). However, I. serusiauxii differs clearly from C. cerina s.l. by the black pycnidium ostioles, the presence of the pigment Cinereorufa-green, and the thick ascospore septa 8-12 $\mu \mathrm{m}$ wide instead of 5-8 $\mu \mathrm{m}$ (Fletcher $\&$ Laundon 2009). Another rather similar species in the Mediterranean, Caloplaca haematites, has, like C. cerina, an anthraquinone-free thallus, anthraquinone-free apothecium margins and often orange-coloured discs, but in full light the thallus is very dark, almost black, due to a different, grey, $\mathrm{K}+$ violet pigment (Sedifolia-grey), and the discs are reddish; only in shade are the thallus greenish grey and the discs orange. Thus, I. serusiauxii is clearly distinct in full light by the thallus- and apothecium color, and by the presence of Cinereorufa-green, while 
Table 1. Preliminary checklist of 221 lichenized fungi known from Porto Santo. Added are habitat $(\mathrm{sax}=$ on rock; ter $=$ on soil; cor $=$ corticolous), herbaria where vouchers are available, availability of TLC results, ITS sequences stored in Genbank, and references to published reports. Pictures of many species as well as some provisionally identified ones can be found on the website https://archive.bgbm.org/sipman/Zschackia/ PortoSanto/genuslist.htm

\begin{tabular}{|c|c|c|c|c|c|}
\hline Taxon & Habitat & Voucher & TLC & ITS sequences & Publications \\
\hline Acarospora lavicola J. Steiner & $\operatorname{sax}$ & $\mathrm{B}, \mathrm{M}$ & - & MN586918 & Sipman \& Aptroot (2019) \\
\hline Acarospora veronensis A. Massal. & sax & $\mathrm{B}, \mathrm{M}$ & - & MN586919 & Sipman \& Aptroot (2019) \\
\hline Acrocordia salweyi (Leight. ex Nyl.) A. L. Sm. & $\operatorname{sax}$ & M & - & - & Sipman \& Aptroot (2019) \\
\hline Agonimia tristicula (Nyl.) Zahlbr. & cor & M & - & - & Sipman \& Aptroot (2019) \\
\hline Alyxoria ochrocheila (Nyl.) Ertz \& Tehler & $\operatorname{sax}$ & M & - & - & Sipman \& Aptroot (2019) \\
\hline Alyxoria varia (Pers.) Ertz \& Tehler & cor & $\mathrm{B}, \mathrm{M}$ & - & - & Sipman \& Aptroot (2019) \\
\hline Amandinea pelidna (Ach.) Fryday \& L. Arcadia & $\operatorname{sax}$ & B & - & - & Sipman \& Aptroot (2019) \\
\hline Amandinea sp. & $\operatorname{sax}$ & B & - & MN586920, MN586921 & Sipman \& Aptroot (2019) \\
\hline Anzia centrifuga Haugan & $\operatorname{sax}$ & $\mathrm{B}, \mathrm{BR}, \mathrm{M}$ & - & - & $\begin{array}{l}\text { Haugan (1992), Sparrius } \\
\text { et al. (2017) }\end{array}$ \\
\hline $\begin{array}{l}\text { Arthonia calcarea (Turner ex Sm.) Ertz } \\
\quad \text { \& Diederich }\end{array}$ & $\operatorname{sax}$ & M & - & - & Sipman \& Aptroot (2019) \\
\hline Arthonia punctiformis Ach. & cor & $\mathrm{B}, \mathrm{M}$ & - & - & Sipman \& Aptroot (2019) \\
\hline Aspiciliella portosantana Sipman \& Zakeri & $\operatorname{sax}$ & $\mathrm{B}, \mathrm{BR}, \mathrm{M}$ & yes & $\begin{array}{l}\text { MN586922, MN586923, } \\
\text { MN586924 }\end{array}$ & Zakeri et al. (2017) \\
\hline Bacidia laurocerasi (Delise ex Duby) Zahlbr. & cor & M & - & - & Sipman \& Aptroot (2019) \\
\hline Bacidina arnoldiana (Körb.) V. Wirth \& Vězda & sax & M & - & - & Sipman \& Aptroot (2019) \\
\hline $\begin{array}{l}\text { Bactrospora thyrsodes (Stirt.) Llop \& Van den } \\
\text { Boom }\end{array}$ & $\operatorname{sax}$ & M & - & - & Sipman \& Aptroot (2019) \\
\hline Blastenia festivella (Nyl.) Vondrák & sax & $\mathrm{B}, \mathrm{M}$ & - & $\begin{array}{l}\text { MN586926, MN586927, } \\
\text { MN586928, MN586929 }\end{array}$ & Sipman \& Aptroot (2019) \\
\hline Buellia caloplacivora Llimona \& Egea & $\operatorname{sax}$ & B & - & - & Sipman \& Aptroot (2019) \\
\hline Buellia dispersa A. Massal. & $\operatorname{sax}$ & & - & - & Sparrius et al. (2017) \\
\hline Buellia mediterranea Giralt & cor & B & - & - & Sipman \& Aptroot (2019) \\
\hline Buellia spuria (Schaer.) Anzi & $\operatorname{sax}$ & $\mathrm{B}, \mathrm{M}$ & yes & MN586930 & Follmann (1990) as B. lactea \\
\hline Buellia stellulata (Taylor) Mudd & $\operatorname{sax}$ & $\mathrm{B}, \mathrm{M}$ & - & - & Sipman \& Aptroot (2019) \\
\hline Buellia tesserata (Taylor) Mudd & sax & B & yes & MN586931, MN586932 & Sipman \& Aptroot (2019) \\
\hline Caloplaca cf. albolutescens (Nyl.) H. Olivier & $\operatorname{sax}$ & $\mathrm{B}, \mathrm{M}$ & - & MN586934 & Sipman \& Aptroot (2019) \\
\hline Caloplaca cf. ceracea J. R. Laundon & sax & $\mathrm{B}, \mathrm{M}$ & - & MN586925, MN586935 & Sipman \& Aptroot (2019) \\
\hline $\begin{array}{l}\text { Caloplaca cf. flavovirescens (Wulfen) Dalla } \\
\text { Torre \& Sarnth. }\end{array}$ & cor & B & - & MN586938, MN586937 & Sipman \& Aptroot (2019) \\
\hline $\begin{array}{l}\text { Caloplaca cf. neotaurica Vondrák, Khodos., } \\
\text { Arup \& Søchting }\end{array}$ & $\operatorname{sax}$ & B & - & $\begin{array}{l}\text { MN586946, MN586933, } \\
\text { MN586947, MN586948 }\end{array}$ & Sipman \& Aptroot (2019) \\
\hline Candelariella vitellina (Hoffm.) Müll. Arg. & $\operatorname{sax}$ & $\mathrm{B}, \mathrm{BR}, \mathrm{M}$ & - & - & Follmann (1990) \\
\hline Catillaria atomarioides (Müll. Arg.) H. Kilias & sax & M & - & - & Sipman \& Aptroot (2019) \\
\hline Catillaria chalybeia (Borrer) A. Massal. & sax & $\mathrm{B}, \mathrm{M}$ & - & - & Sipman \& Aptroot (2019) \\
\hline Catillaria mediterranea Hafellner & $\begin{array}{l}\text { Licheni- } \\
\text { cilous }\end{array}$ & B & - & - & $\begin{array}{l}\text { Sipman \& Aptroot (2019) on } \\
\text { Ramalina crispatula, lower } \\
\text { part of lobes }\end{array}$ \\
\hline Catillaria minuta (Schaer.) Lettau & sax & M & - & - & Sipman \& Aptroot (2019) \\
\hline Chaenotheca furfuracea (L.) Tibell & cor & M & - & - & Sipman \& Aptroot (2019) \\
\hline Chrysothrix candelaris (L.) J. R. Laundon & sax, ter, cor & M & - & - & Sipman \& Aptroot (2019) \\
\hline $\begin{array}{l}\text { Circinaria contorta (Hoffm.) A. Nordin, Savić } \\
\quad \& \text { Tibell }\end{array}$ & $\operatorname{sax}$ & $\mathrm{B}, \mathrm{M}$ & - & - & Sipman \& Aptroot (2019) \\
\hline Cladonia humilis (With.) J. R. Laundon & ter & $\mathrm{B}, \mathrm{M}$ & - & - & Sipman \& Aptroot (2019) \\
\hline Cladonia macilenta Hoffm. & ter & M & - & - & Sipman \& Aptroot (2019) \\
\hline Cladonia microphylla Ahti \& Aptroot & ter & $\mathrm{B}, \mathrm{M}$ & yes & MN586949 & Sipman \& Aptroot (2019) \\
\hline Cladonia peziziformis (With.) J. R. Laundon & ter & $\mathrm{B}, \mathrm{M}$ & yes & - & Sipman \& Aptroot (2019) \\
\hline Cladonia ramulosa (With.) J. R. Laundon & ter & M & - & - & Sipman \& Aptroot (2019) \\
\hline Cladonia rangiformis Hoffm. & ter & $\mathrm{B}, \mathrm{M}$ & yes & - & Sipman \& Aptroot (2019) \\
\hline Cladonia stereoclada Abbayes & ter & $\mathrm{B}, \mathrm{M}$ & - & MN586950 & Sipman \& Aptroot (2019) \\
\hline Clauzadea metzleri (Körb.) & sax & M & - & - & $\begin{array}{l}\text { Meyer (2002), Clauzade } \\
\& \text { Cl. Roux ex D. Hawksw. }\end{array}$ \\
\hline Clavascidium lacinulatum (Ach.) M. Prieto & ter & $\mathrm{BR}, \mathrm{M}$ & - & - & Sipman \& Aptroot (2019) \\
\hline Cliostomum griffithii (Sm.) Coppins & sax, cor & $\mathrm{B}, \mathrm{BR}, \mathrm{M}$ & - & - & Sipman \& Aptroot (2019) \\
\hline $\begin{array}{l}\text { Coccocarpia erythroxyli (Spreng.) Swinscow } \\
\quad \& \text { Krog }\end{array}$ & $\operatorname{sax}$ & M & - & - & Sipman \& Aptroot (2019) \\
\hline Coenogonium luteum (Dicks.) Kalb \& Lücking & cor & M & - & - & Sipman \& Aptroot (2019) \\
\hline
\end{tabular}


Table 1. Continued.

\begin{tabular}{|c|c|c|c|c|c|}
\hline Taxon & Habitat & Voucher & TLC & ITS sequences & Publications \\
\hline Collema ryssoleum (Tuck.) A. Schneid. & sax & $\mathrm{B}, \mathrm{M}$ & - & - & Sipman \& Aptroot (2019) \\
\hline $\begin{array}{l}\text { Collemopsidium caesium (Nyl.) Coppins } \\
\text { \& Aptroot }\end{array}$ & $\operatorname{sax}$ & M & - & - & Sipman \& Aptroot (2019) \\
\hline Cresponea premnea (Ach.) Egea \& Torrente & cor & $\mathrm{B}, \mathrm{M}$ & - & - & Sipman \& Aptroot (2019) \\
\hline Crocodia aurata (Ach.) Link & cor & $\mathrm{B}, \mathrm{M}$ & - & - & Sipman \& Aptroot (2019) \\
\hline Dimelaena radiata (Tuck.) Hale \& W. L. Culb. & & B & yes & - & Sipman \& Aptroot (2019) \\
\hline Diploicia canescens (Dicks.) A. Massal. & sax & $\mathrm{B}, \mathrm{BR}, \mathrm{M}$ & - & - & Sipman \& Aptroot (2019) \\
\hline $\begin{array}{l}\text { Diploicia subcanescens (Werner) Hafellner } \\
\quad \text { \& Poelt }\end{array}$ & sax & $\mathrm{B}, \mathrm{BR}, \mathrm{M}$ & - & - & Follmann (1990) \\
\hline Diploschistes actinostomus (Ach.) Zahlbr. & $\operatorname{sax}$ & $\mathrm{B}, \mathrm{M}$ & - & $\begin{array}{l}\text { MN586951, MN586952, } \\
\text { MN586953 }\end{array}$ & $\begin{array}{l}\text { Follmann }(1990) \text { as } \\
\text { D. caesioplumbeus }\end{array}$ \\
\hline Dirina cf. ceratoniae (Ach.) Fr. & cor & - & - & - & $\begin{array}{l}\text { Follmann (1990), Sparrius } \\
\text { et al. (2017) }\end{array}$ \\
\hline Dirina insulana (Tav.) Tehler & sax & $\mathrm{B}, \mathrm{BR}, \mathrm{M}$ & - & - & $\begin{array}{l}\text { Tehler et al. (2013), Foll- } \\
\text { mann (1990) as D. massil- } \\
\text { iensis }\end{array}$ \\
\hline $\begin{array}{l}\text { Dirina paradoxa subsp. africana (Tehler) } \\
\text { Tehler }\end{array}$ & cor & - & - & - & Sipman \& Aptroot (2019) \\
\hline Dirinaria applanata (Fée) D. D. Awasthi & $\operatorname{sax}$ & B & - & MN586954 & Sipman \& Aptroot (2019) \\
\hline Enchylium tenax (Sw.) Gray & ter & $\mathrm{BR}, \mathrm{M}$ & - & - & Sipman \& Aptroot (2019) \\
\hline Endocarpon pusillum Hedw. & ter & B & - & - & Sipman \& Aptroot (2019) \\
\hline $\begin{array}{l}\text { Endohyalina ericina (Nyl.) Giralt, van den } \\
\text { Boom \& Elix }\end{array}$ & cor & $\mathrm{B}, \mathrm{M}$ & - & MN587027, MN587028 & Sipman \& Aptroot (2019) \\
\hline Enterographa hutchinsiae (Leight.) A. Massal. & sax & B & - & - & Sipman \& Aptroot (2019) \\
\hline Epiphloea terrena (Nyl.) Trevis. & ter & $\mathrm{B}, \mathrm{M}$ & - & - & Sipman \& Aptroot (2019) \\
\hline $\begin{array}{l}\text { Flavoplaca } \text { cf. maritima (B. de Lesd.) Arup, } \\
\text { Frödén \& Søchting }\end{array}$ & $\operatorname{sax}$ & B & - & $\begin{array}{l}\text { MN586941, MN586939, } \\
\text { MN586940, MN586936, } \\
\text { MN586942, MN586943, } \\
\text { MN586944, MN586945 }\end{array}$ & Sipman \& Aptroot (2019) \\
\hline Fulgensia desertorum (Tomin) Poelt & ter & & - & - & Follmann (1990) \\
\hline $\begin{array}{l}\text { Fulvophyton sorediatum (Sparrius, P. James } \\
\quad \& \text { M. A. Allen) Tehler \& van den Boom }\end{array}$ & $\operatorname{sax}$ & $\mathrm{B}, \mathrm{M}$ & - & - & Sparrius et al. (2017) \\
\hline Gyalecta schisticola Werner & sax, ter & $\mathrm{B}, \mathrm{M}$ & - & - & Sipman \& Aptroot (2019) \\
\hline $\begin{array}{l}\text { Haloplaca sorediella (Arup) Arup, Frödén } \\
\quad \& \text { Søchting }\end{array}$ & sax, cor & B & - & MN586955 & Sipman \& Aptroot (2019) \\
\hline Heppia conchiloba Werner & ter & M & - & - & Sipman \& Aptroot (2019) \\
\hline Heterodermia leucomelos (L.) Poelt & sax, ter, cor & $\mathrm{B}, \mathrm{BR}, \mathrm{M}$ & yes & - & Carvalho et al. (2008) \\
\hline $\begin{array}{l}\text { Hyperphyscia adglutinata (Flörke) } \\
\text { H. Mayrhofer \& Poelt }\end{array}$ & cor & BR & - & - & Sipman \& Aptroot (2019) \\
\hline Ikaeria serusiauxii Sipman & cor & B & - & $\begin{array}{l}\text { MN586958, MN586959, } \\
\text { MN586960 }\end{array}$ & Sipman \& Aptroot (2019) \\
\hline $\begin{array}{l}\text { Ikaeria aurantiellina (Harm.) S. Y. Kondr., } \\
\text { Upreti \& Hur (syn. Caloplaca aegatica } \\
\text { Giralt, Nimis \& Poelt) }\end{array}$ & cor & B & - & MN586957, MN586956 & Sipman \& Aptroot (2019) \\
\hline $\begin{array}{l}\text { Lecania cuprea (A. Massal.) Van den Boom } \\
\quad \text { \& Coppins }\end{array}$ & sax & M & - & - & Sipman \& Aptroot (2019) \\
\hline $\begin{array}{l}\text { Lecania naegelii (Hepp) Diederich \& Van den } \\
\quad \text { Boom }\end{array}$ & cor & M & - & - & Sipman \& Aptroot (2019) \\
\hline Lecania nigra van den Boom \& Ertz & sax & B & - & - & Sipman \& Aptroot (2019) \\
\hline Lecania sylvestris (Arnold) Arnold & sax & $\mathrm{M}$ & - & - & Sipman \& Aptroot (2019) \\
\hline Lecania turicensis (Hepp) Müll. Arg. & $\operatorname{sax}$ & BR, M & - & - & Sipman \& Aptroot (2019) \\
\hline $\begin{array}{l}\text { Lecanographa dialeuca (Cromb.) Egea } \\
\quad \text { \& Torrente }\end{array}$ & sax & $\mathrm{B}, \mathrm{BR}, \mathrm{M}$ & - & - & Sparrius et al. (2017) \\
\hline Lecanora campestris (Schaer.) Hue & sax & M & yes & MN586965 & Sipman \& Aptroot (2019) \\
\hline Lecanora confusa Almb. & cor & $\mathrm{B}, \mathrm{M}$ & & - & Follmann (1990) \\
\hline Lecanora gangaleoides Nyl. & sax & M & yes & - & Sipman \& Aptroot (2019) \\
\hline Lecanora cf. hybocarpa (Tuck.) Brodo & cor & $\mathrm{B}, \mathrm{M}$ & yes & $\begin{array}{l}\text { MN586968, MN586969, } \\
\text { MN586970 }\end{array}$ & Sipman \& Aptroot (2019) \\
\hline $\begin{array}{l}\text { Lecanora cf. oreinoides (Körb.) Hertel } \\
\quad \text { \& Rambold }\end{array}$ & $\operatorname{sax}$ & B & yes & MN586966, MN586967 & Sipman \& Aptroot (2019) \\
\hline Lecanora cf. praepostera Nyl. & $\operatorname{sax}$ & $\mathrm{B}, \mathrm{M}$ & yes & $\begin{array}{l}\text { MN586972, MN586971, } \\
\text { MN586973 }\end{array}$ & Sipman \& Aptroot (2019) \\
\hline Lecanora sulphurella Hepp & sax & $\mathrm{B}, \mathrm{BR}, \mathrm{M}$ & - & - & Follmann (1990) \\
\hline Lecanora sp. 1 & $\operatorname{sax}$ & B & yes & MN586961, MN586962 & Sipman \& Aptroot (2019) \\
\hline
\end{tabular}


Table 1. Continued.

\begin{tabular}{|c|c|c|c|c|c|}
\hline Taxon & Habitat & Voucher & TLC & ITS sequences & Publications \\
\hline Lecanora sp. 2 & sax & B & yes & MN586974 & Sipman \& Aptroot (2019) \\
\hline Lecanora sp. 3 & sax & $\mathrm{B}, \mathrm{M}$ & yes & MN586963, MN586964 & Sipman \& Aptroot (2019) \\
\hline Lecidea sarcogynoides Körb. & sax & $\mathrm{B}, \mathrm{BR}, \mathrm{M}$ & - & - & Sipman \& Aptroot (2019) \\
\hline $\begin{array}{l}\text { Lecidella } \mathrm{cf.} \text { elaeochromoides (Nyl.) Knoph } \\
\quad \& \text { Hertel }\end{array}$ & sax & B & - & MN586975, MN586976 & Sipman \& Aptroot (2019) \\
\hline $\begin{array}{l}\text { Lecidella } \text { cf. meiococca (Nyl.) Leuckert } \\
\quad \& \text { Hertel }\end{array}$ & sax & B & - & MN586979 & Sipman \& Aptroot (2019) \\
\hline Lecidella scabra (Taylor) Hertel \& Leuckert & sax & M & - & - & Sipman \& Aptroot (2019) \\
\hline Lecidella $\mathrm{sp}$. & $\operatorname{sax}$ & B & - & MN586977, MN586978 & Sipman \& Aptroot (2019) \\
\hline Lepra amara (Ach.) Hafellner & cor & B & yes & - & Sipman \& Aptroot (2019) \\
\hline Lepra corallina (L.) Hafellner & - & $\begin{array}{l}\text { photo } \\
\text { M. Vervoort }\end{array}$ & - & - & Sipman \& Aptroot (2019) \\
\hline Lepra monogona (Nyl.) Hafellner & $\operatorname{sax}$ & $\begin{array}{l}\text { photo } \\
\text { M. Vervoort }\end{array}$ & - & - & Sipman \& Aptroot (2019) \\
\hline Lepra teneriffensis (Vain.) Hafellner & sax & $\mathrm{B}, \mathrm{M}$ & yes & - & $\begin{array}{l}\text { Sparrius et al. (2017) as } \\
\text { Pertusaria excludens }\end{array}$ \\
\hline $\begin{array}{l}\text { Lepra trachythallina (Erichsen) Lendemer } \\
\text { \& R. C. Harris }\end{array}$ & cor & $\mathrm{B}, \mathrm{M}$ & yes & - & Sipman \& Aptroot (2019) \\
\hline Lepraria maderensis Kukwa \& Flakus & sax & B & yes & - & Sipman \& Aptroot (2019) \\
\hline Leprocaulon microscopicum (Vill.) Gams & sax, ter & $\mathrm{B}, \mathrm{M}$ & - & - & Sipman \& Aptroot (2019) \\
\hline $\begin{array}{l}\text { Leptogium teretiusculum (Flörke ex Wallr.) } \\
\text { Arnold }\end{array}$ & sax, ter & $\mathrm{B}, \mathrm{M}$ & - & - & Sipman \& Aptroot (2019) \\
\hline Lobaria macaronesica C. Cornejo \& Scheid. & sax & $\mathrm{B}, \mathrm{M}$ & - & - & $\begin{array}{l}\text { Carvalho et al. (2008) as } \\
\text { L. pulmonaria }\end{array}$ \\
\hline $\begin{array}{l}\text { Lobothallia recedens (Taylor) A. Nordin, Savić } \\
\quad \& \text { Tibell }\end{array}$ & $\begin{array}{l}\text { sax on } \\
\text { Aspiciliella } \\
\text { portosan- } \\
\text { tana }\end{array}$ & B & - & MN586980 & Sipman \& Aptroot (2019) \\
\hline Mycoporum sparsellum Nyl. & cor & M & - & - & Sipman \& Aptroot (2019) \\
\hline $\begin{array}{l}\text { Myriolecis crenulata (Ach.) Śliwa, Zhao Xin } \\
\quad \text { \& Lumbsch }\end{array}$ & sax & BR & - & - & Sipman \& Aptroot (2019) \\
\hline $\begin{array}{l}\text { Myriolecis dispersa (Pers.) Śliwa, Zhao Xin } \\
\quad \& \text { Lumbsch }\end{array}$ & sax & $\mathrm{B}, \mathrm{M}$ & - & - & Sipman \& Aptroot (2019) \\
\hline $\begin{array}{l}\text { Myriolecis hagenii (Ach.) Śliwa, Zhao Xin } \\
\quad \& \text { Lumbsch }\end{array}$ & sax & $\mathrm{BR}, \mathrm{M}$ & - & - & Sipman \& Aptroot (2019) \\
\hline Nephroma foliolatum P. James \& F. J. White & sax, ter & M & - & - & Sipman \& Aptroot (2019) \\
\hline Nephroma laevigatum Ach. & ter & B & - & - & Sipman \& Aptroot (2019) \\
\hline Normandina pulchella (Borrer) Nyl. & sax, cor & M & - & - & Sipman \& Aptroot (2019) \\
\hline $\begin{array}{l}\text { Ochrolechia incarnata (Leight.) Kukwa, } \\
\text { Schmitt \& Ertz }\end{array}$ & sax & $\mathrm{B}, \mathrm{BR}, \mathrm{M}$ & - & MN586981 & Kukwa et al. (2018) \\
\hline Opegrapha demutata Nyl. & sax & M & - & - & Sipman \& Aptroot (2019) \\
\hline Opegrapha lutulenta Nyl. & sax & B & - & - & Sipman \& Aptroot (2019) \\
\hline Opegrapha vulgata (Ach.) Ach. & cor & $\mathrm{B}, \mathrm{BR}, \mathrm{M}$ & - & - & Sipman \& Aptroot (2019) \\
\hline Orcularia insperata (Nyl.) Kalb \& Giralt & cor & $\mathrm{BR}, \mathrm{M}$ & - & - & Sipman \& Aptroot (2019) \\
\hline Pannaria rubiginosa (Thunb. ex Ach.) Delise & cor & M & - & - & Sipman \& Aptroot (2019) \\
\hline Pannaria tavaresii P. M. Jørg. & ter, cor & $\mathrm{BR}, \mathrm{M}$ & - & - & Sipman \& Aptroot (2019) \\
\hline $\begin{array}{l}\text { Paralecanographa grumulosa (Dufour) Ertz } \\
\quad \& \text { Tehler }\end{array}$ & sax, lich & B & - & - & Sipman \& Aptroot (2019) \\
\hline Parmotrema perlatum (Huds.) M. Choisy & sax, cor & $\mathrm{B}, \mathrm{M}$ & yes & - & $\begin{array}{l}\text { Carvalho et al. (2008) as } \\
P \text {. chinense }\end{array}$ \\
\hline Parmotrema reticulatum (Taylor) M. Choisy & sax, cor & $\mathrm{B}, \mathrm{BR}, \mathrm{M}$ & yes & - & $\begin{array}{l}\text { Carvalho et al. }(2008) \text { as } \\
\text { Rimelia cetrata }\end{array}$ \\
\hline Parmotrema tinctorum (Despr. ex Nyl.) Hale & sax, cor & $\mathrm{B}, \mathrm{BR}, \mathrm{M}$ & - & - & Sparrius et al. (2017) \\
\hline $\begin{array}{l}\text { Pectenia atlantica (Degel.) P. M. Jørg., } \\
\text { L. Lindblom, Wedin \& S. Ekman }\end{array}$ & sax, ter, cor & $\mathrm{B}, \mathrm{BR}, \mathrm{M}$ & - & - & Sipman \& Aptroot (2019) \\
\hline Peltula bolanderi (Tuck.) Wetmore & $\operatorname{sax}$ & M & - & - & Sipman \& Aptroot (2019) \\
\hline Peltula euploca (Ach.) Poelt & sax & $\mathrm{B}, \mathrm{BR}, \mathrm{M}$ & - & - & Sipman \& Aptroot (2019) \\
\hline Peltula obscurans (Nyl.) Gyeln. & sax, ter & $\mathrm{B}, \mathrm{M}$ & - & - & Sipman \& Aptroot (2019) \\
\hline Peltula omphaliza (Nyl.) Wetmore & sax & M & - & - & Sipman \& Aptroot (2019) \\
\hline Pertusaria aleianta Nyl. & sax & $\mathrm{B}, \mathrm{BR}, \mathrm{M}$ & yes & - & $\begin{array}{l}\text { Follmann }(1990) \text { as } \\
P . \text { gallica, Sparrius et al. } \\
(2017) \text { as } P \text {. pluripuncta }\end{array}$ \\
\hline Pertusaria heterochroa (Müll. Arg.) Erichsen & cor & B & yes & - & Sipman \& Aptroot (2019) \\
\hline
\end{tabular}


Table 1. Continued.

\begin{tabular}{|c|c|c|c|c|c|}
\hline Taxon & Habitat & Voucher & TLC & ITS sequences & Publications \\
\hline Physcia adscendens (Fr.) H. Olivier & cor & $\mathrm{B}$ & & - & Sipman \& Aptroot (2019) \\
\hline Physcia erumpens Moberg & $\operatorname{sax}$ & $\mathrm{B}, \mathrm{M}$ & - & - & Sipman \& Aptroot (2019) \\
\hline Placidium boccanum (Servít) Breuss & sax & $\mathrm{BR}, \mathrm{M}$ & - & - & Sipman \& Aptroot (2019) \\
\hline Placidium squamulosum (Ach.) Breuss & ter & $\mathrm{BR}, \mathrm{M}$ & - & - & Sipman \& Aptroot (2019) \\
\hline Placynthiella dasaea (Stirt.) Tønsberg & cor & M & - & - & Sipman \& Aptroot (2019) \\
\hline Placynthium nigrum (Huds.) Gray & $\operatorname{sax}$ & M & - & - & Sipman \& Aptroot (2019) \\
\hline Polysporina cyclocarpa (Anzi) Vězda & sax, ter & M & - & - & Sipman \& Aptroot (2019) \\
\hline Polysporina simplex (Taylor) Vězda & sax & B & - & - & Sipman \& Aptroot (2019) \\
\hline Porina curnowii A. L. Sm. & sax & $\mathrm{B}, \mathrm{M}$ & - & - & Sipman \& Aptroot (2019) \\
\hline Porina leptospora (Nyl.) A. L. Sm. & cor & $\mathrm{B}$ & - & - & Sipman \& Aptroot (2019) \\
\hline $\begin{array}{l}\text { Porpidia albocoerulescens (Wulfen) Hertel } \\
\quad \text { \& Knoph }\end{array}$ & sax & M & - & - & Sipman \& Aptroot (2019) \\
\hline Porpidia crustulata (Ach.) Hertel \& Knoph & $\operatorname{sax}$ & M & - & - & Sipman \& Aptroot (2019) \\
\hline $\begin{array}{l}\text { Protoparmelia montagnei (Fr.) Sancho } \\
\quad \text { \& A. Crespo }\end{array}$ & sax & $\mathrm{B}, \mathrm{M}$ & - & MN586983, MN586982 & Sipman \& Aptroot (2019) \\
\hline Protoparmeliopsis muralis (Schreb.) M. Choisy & sax & BR & - & - & Sipman \& Aptroot (2019) \\
\hline Psora decipiens (Hedw.) Hoffm. & ter & & - & - & Follmann (1990) \\
\hline Psorotichia murorum A. Massal. & $\operatorname{sax}$ & M & - & - & Sipman \& Aptroot (2019) \\
\hline Pyrenula chlorospila (Nyl.) Arnold & cor & M & - & - & Sipman \& Aptroot (2019) \\
\hline Pyrrhospora quernea (Dicks.) Körb. & cor & $\mathrm{B}, \mathrm{M}$ & - & - & Sipman \& Aptroot (2019) \\
\hline Pyxine sorediata (Ach.) Mont. & $\operatorname{sax}$ & $\mathrm{B}, \mathrm{M}$ & - & - & Sipman \& Aptroot (2019) \\
\hline Pyxine subcinerea Stirt. & sax & M & - & - & Sipman \& Aptroot (2019) \\
\hline Ramalina canariensis J. Steiner & cor & B & yes & - & Sipman \& Aptroot (2019) \\
\hline Ramalina chondrina J. Steiner & cor & $\mathrm{B}, \mathrm{M}$ & yes & - & Sipman \& Aptroot (2019) \\
\hline Ramalina confertula Krog \& Østh. & sax, cor & $\mathrm{B}, \mathrm{M}$ & yes & - & $\begin{array}{l}\text { Krog \& Østhagen (1980), } \\
\text { Follmann (1990), Sparrius } \\
\text { et al. (2017) }\end{array}$ \\
\hline Ramalina crispatula Despr. ex Nyl. & $\operatorname{sax}$ & $\mathrm{B}, \mathrm{M}$ & yes & MN586989 & $\begin{array}{l}\text { Follmann (1990), Sparrius } \\
\text { et al. }(2017)\end{array}$ \\
\hline Ramalina decipiens Mont. & $\operatorname{sax}$ & $\mathrm{B}, \mathrm{M}$ & yes & $\begin{array}{l}\text { MN586991, MN586990, } \\
\text { MN586993, MN586992, } \\
\text { MN586994 }\end{array}$ & $\begin{array}{l}\text { Sérusiaux et al. (2010) as } \\
R . \text { subwebbiana, Sparrius } \\
\text { et al. ( } 2017)\end{array}$ \\
\hline Ramalina erosa Krog & sax & M & yes & $\begin{array}{l}\text { MN586995, MN586996, } \\
\text { MN586997 }\end{array}$ & $\begin{array}{l}\text { Krog (1990), Sérusiaux et al. } \\
(2010) \text {, Sparrius et al. (2017) }\end{array}$ \\
\hline Ramalina fastigiata (Pers.) Ach. & cor & $\mathrm{B}, \mathrm{M}$ & yes & MN586998, MN586999 & Sipman \& Aptroot (2019) \\
\hline Ramalina huei Harm. & cor & $\mathrm{B}, \mathrm{M}$ & yes & MN587000, MN587001 & Sipman \& Aptroot (2019) \\
\hline Ramalina jamesii Krog & $\operatorname{sax}$ & $\mathrm{B}, \mathrm{M}$ & yes & MN587002, MN587003 & $\begin{array}{l}\text { Krog (1990), Sparrius et al. } \\
(2017)\end{array}$ \\
\hline Ramalina lacera (With.) J. R. Laundon & cor & $\mathrm{B}, \mathrm{M}$ & yes & - & Follmann (1990) as R. duriaei \\
\hline Ramalina maderensis Motyka & $\operatorname{sax}$ & $\mathrm{B}, \mathrm{M}$ & yes & $\begin{array}{l}\text { MN587004, MN587005, } \\
\text { MN587006 }\end{array}$ & $\begin{array}{l}\text { Follmann (1990), Sérusiaux } \\
\text { et al. (2010) }\end{array}$ \\
\hline Ramalina cf. maderensis (divaricatic acid) & $\operatorname{sax}$ & B & yes & $\begin{array}{l}\text { MN586988, MN586984, } \\
\text { MN586985, MN586986, } \\
\text { MN586987 }\end{array}$ & Sipman \& Aptroot (2019) \\
\hline Ramalina mollis Krog & cor & $\mathrm{B}, \mathrm{M}$ & yes & - & Sipman \& Aptroot (2019) \\
\hline Ramalina nematodes (Nyl.) Krog \& Østh. & sax, cor & $\mathrm{B}, \mathrm{BR}, \mathrm{M}$ & yes & MN587007, MN587008 & $\begin{array}{l}\text { Krog \& Østhagen (1980), } \\
\text { Follmann (1990), Sparrius } \\
\text { et al. (2017) }\end{array}$ \\
\hline Ramalina portosantana $\mathrm{Krog}$ & $\operatorname{sax}$ & M & - & - & $\begin{array}{l}\text { Krog (1990), Sérusiaux et al. } \\
(2010) \text {, Sparrius et al. (2017) }\end{array}$ \\
\hline Ramalina pusilla Le Prévost & cor & $\mathrm{B}, \mathrm{M}$ & - & - & Sipman \& Aptroot (2019) \\
\hline Ramalina requienii (De Not.) Jatta & sax, cor & $\mathrm{B}, \mathrm{M}$ & yes & $\begin{array}{l}\text { MN587009, MN587010, } \\
\text { MN587011, MN587012, } \\
\text { MN587013, MN587014, } \\
\text { MN587015, MN587016 }\end{array}$ & $\begin{array}{l}\text { Carvalho et al. (2008) as } \\
R \text {. polymorpha, Sparrius } \\
\text { et al. (2017) }\end{array}$ \\
\hline Ramalina subpusilla (Nyl.) Zahlbr. & cor & $\mathrm{B}, \mathrm{M}$ & yes & $\begin{array}{l}\text { MN587017, MN587018, } \\
\text { MN587019 }\end{array}$ & Sipman \& Aptroot (2019) \\
\hline Ramalina timdaliana $\mathrm{Krog}$ & $\operatorname{sax}$ & M & - & - & $\begin{array}{l}\text { Krog (1990), Sparrius et al. } \\
\text { (2017) }\end{array}$ \\
\hline Ramalina tingitana Salzm. & sax, cor & $\mathrm{B}, \mathrm{M}$ & yes & $\begin{array}{l}\text { MN587025, MN587020, } \\
\text { MN587021, MN587022, } \\
\text { MN587023, MN587024 }\end{array}$ & $\begin{array}{l}\text { Follmann (1990) also } \\
\text { as } R \text {. bourgeana; Krog } \\
\& \text { Østhagen (1980), Sparrius } \\
\text { et al. (2017) }\end{array}$ \\
\hline
\end{tabular}


Table 1. Continued.

\begin{tabular}{|c|c|c|c|c|c|}
\hline Taxon & Habitat & Voucher & TLC & ITS sequences & Publications \\
\hline Rhizocarpon lusitanicum (Nyl.) Arnold & sax & $\mathrm{B}, \mathrm{M}$ & - & - & Sipman \& Aptroot (2019) \\
\hline Rhymbocarpus boomii Etayo \& Diederich & $\begin{array}{l}\text { lichenico- } \\
\text { lous }\end{array}$ & B & - & - & $\begin{array}{l}\text { Sipman \& Aptroot (2019) on } \\
\text { Roccella phycopsis }\end{array}$ \\
\hline $\begin{array}{l}\text { Rinodina anomala (Zahlbr.) H. Mayrhofer } \\
\quad \& \text { Giralt }\end{array}$ & cor & B, BR, M & - & MN587026, MN587027 & Sipman \& Aptroot (2019) \\
\hline Rinodina cf. anomala & cor & B & - & MN587028 & Sipman \& Aptroot (2019) \\
\hline Rinodina beccariana Bagl. & $\operatorname{sax}$ & M & - & - & Sipman \& Aptroot (2019) \\
\hline $\begin{array}{l}\text { Rinodina beccariana var. lavicola (J. Steiner) } \\
\text { Matzer \& H. Mayrhofer }\end{array}$ & $\operatorname{sax}$ & $\mathrm{B}, \mathrm{M}$ & - & - & Sipman \& Aptroot (2019) \\
\hline Rinodina cana (Arnold) Arnold & $\operatorname{sax}$ & B & - & MN587029 & Sipman \& Aptroot (2019) \\
\hline $\begin{array}{l}\text { Rinodina canariensis Matzer, H. Mayrhofer } \\
\quad \& \text { P. Clerc }\end{array}$ & $\operatorname{sax}$ & $\mathrm{BR}, \mathrm{M}$ & - & - & Sipman \& Aptroot (2019) \\
\hline Rinodina exigua (Ach.) Gray & cor & M & - & - & Sipman \& Aptroot (2019) \\
\hline Rinodina immersa (Körb.) J. Steiner & $\operatorname{sax}$ & M & - & - & Sipman \& Aptroot (2019) \\
\hline Rinodina intermedia Bagl. & ter & B & - & - & Sipman \& Aptroot (2019) \\
\hline Rinodina oleae Bagl. & $\operatorname{sax}$ & M & - & - & Sipman \& Aptroot (2019) \\
\hline Rinodina oxydata (A. Massal.) A. Massal. & $\operatorname{sax}$ & B & - & - & Sipman \& Aptroot (2019) \\
\hline Rinodina pruinella Bagl. & cor & B & yes & - & Sipman \& Aptroot (2019) \\
\hline Roccella allorgei Abbayes & $\operatorname{sax}$ & M & - & - & Sparrius et al. (2017) \\
\hline Roccella elisabethae Tehler & $\operatorname{sax}$ & & - & - & Tehler et al. (2004) \\
\hline Roccella fuciformis (L.) DC. & $\operatorname{sax}$ & $\mathrm{B}, \mathrm{BR}, \mathrm{M}$ & - & - & Sipman \& Aptroot (2019) \\
\hline Roccella maderensis (J. Steiner) Follmann & $\operatorname{sax}$ & $\mathrm{B}, \mathrm{BR}, \mathrm{M}$ & - & - & Sparrius et al. (2017) \\
\hline Roccella phycopsis Ach. & sax & $\mathrm{B}, \mathrm{BR}, \mathrm{M}$ & - & - & $\begin{array}{l}\text { Follmann }(1990) \text { also as } \\
R \text {. hypomecha }\end{array}$ \\
\hline Roccella tinctoria DC. & $\operatorname{sax}$ & $\mathrm{B}, \mathrm{M}$ & - & - & $\begin{array}{l}\text { Follmann (1990) as } R . \text { tuber- } \\
\text { culata, Carvalho et al. (2008) } \\
\text { as } R . \text { vicentina, Sparrius } \\
\text { et al. (2017) }\end{array}$ \\
\hline $\begin{array}{l}\text { Roccellographa circumscripta (Leight.) Ertz } \\
\quad \& \text { Tehler }\end{array}$ & $\operatorname{sax}$ & M & - & - & Sipman \& Aptroot (2019) \\
\hline $\begin{array}{l}\text { Rufoplaca arenaria (Pers.) Arup, Søchting } \\
\text { \& Frödén }\end{array}$ & sax, ter & B, BR & - & MN587030 & Sparrius et al. (2017) cf. \\
\hline $\begin{array}{l}\text { Rusavskia resendei (Poelt \& Tav.) S. Y. Kondr. } \\
\text { \& Kärnefelt }\end{array}$ & $\operatorname{sax}$ & $\mathrm{B}, \mathrm{BR}, \mathrm{M}$ & - & - & $\begin{array}{l}\text { Carvalho et al. (2008), also } \\
\text { as X. elegans }\end{array}$ \\
\hline Schismatomma albocinctum (Nyl.) Zahlbr. & cor & B & - & - & Sipman \& Aptroot (2019) \\
\hline Schismatomma graphidioides (Leight.) Zahlbr. & cor & $\mathrm{B}, \mathrm{M}$ & - & - & Sipman \& Aptroot (2019) \\
\hline Scoliciosporum umbrinum (Ach.) Arnold & $\operatorname{sax}$ & $\mathrm{B}, \mathrm{M}$ & - & - & Sipman \& Aptroot (2019) \\
\hline $\begin{array}{l}\text { Scytinium aragonii (Otálora) Otálora, } \\
\text { P. M. Jørg. \& Wedin }\end{array}$ & ter & M & - & - & Sipman \& Aptroot (2019) \\
\hline Solenopsora vulturiensis A. Massal. & sax, ter & M & - & - & Sipman \& Aptroot (2019) \\
\hline Sphinctrina tubiformis A. Massal. & $\begin{array}{l}\text { lichenico- } \\
\text { lous }\end{array}$ & B & - & - & $\begin{array}{l}\text { Sipman \& Aptroot (2019) on } \\
\text { Pertusaria heterochroa }\end{array}$ \\
\hline Sphinctrina turbinata (Pers.) De Not. & cor & BR & - & - & Sipman \& Aptroot (2019) \\
\hline Squamarina cartilaginea (With.) P. James & sax, ter & $\mathrm{B}, \mathrm{M}$ & - & - & Sipman \& Aptroot (2019) \\
\hline Syncesia myrticola (Fée) Tehler & sax, cor & $\mathrm{B}, \mathrm{BR}, \mathrm{M}$ & - & MN587031, MN587032 & Sipman \& Aptroot (2019) \\
\hline $\begin{array}{l}\text { Tephromela atra var. deplanata (J. Steiner) } \\
\text { Hafellner \& Hierze }\end{array}$ & $\operatorname{sax}$ & $\mathrm{B}, \mathrm{M}$ & yes & MN587033, MN587034 & Sipman \& Aptroot (2019) \\
\hline Thalloidima albilabrum (Dufour) Flagey & - & - & - & - & Timdal (1992) as Toninia \\
\hline $\begin{array}{l}\text { Thalloidima massatum (Tuck.) Kistenich, } \\
\text { Timdal, Bendiksby \& S.Ekman }\end{array}$ & - & - & - & - & Timdal (1992) as Toninia \\
\hline Thalloidima toepfferi Stein & ter & $\begin{array}{l}\text { photo } \\
\text { M. Vervoort }\end{array}$ & - & - & Sipman \& Aptroot (2019) \\
\hline Thelenella muscorum (Th. Fr.) Vain. & ter & M & - & - & Sipman \& Aptroot (2019) \\
\hline Thelomma mammosum (Hepp) A. Massal. & $\operatorname{sax}$ & $\mathrm{B}, \mathrm{M}$ & yes & - & Sipman \& Aptroot (2019) \\
\hline Thelopsis isiaca Stizenb. & sax & M & - & - & Sipman \& Aptroot (2019) \\
\hline Thelotrema laurisilvae Lücking \& Breuss & cor & $\mathrm{B}, \mathrm{BR}, \mathrm{M}$ & - & - & Sipman \& Aptroot (2019) \\
\hline Toninia plumbina (Anzi) Hafellner \& Timdal & sax & M & - & - & Sipman \& Aptroot (2019) \\
\hline $\begin{array}{l}\text { Toniniopsis aromatica }(\mathrm{Sm} .) \text { Kistenich, Timdal, } \\
\text { Bendiksby \& S. Ekman }\end{array}$ & sax, ter & $\mathrm{B}, \mathrm{BR}, \mathrm{M}$ & - & - & Timdal (1992) as Toninia \\
\hline Toniniopsis mesoidea (Nyl.) Timdal & sax, ter & $\mathrm{B}, \mathrm{BR}, \mathrm{M}$ & - & - & Sipman \& Aptroot (2019) \\
\hline Trapelia coarctata (Turner) M. Choisy & ter & M & - & - & Sipman \& Aptroot (2019) \\
\hline
\end{tabular}


Table 1. Continued.

\begin{tabular}{|c|c|c|c|c|c|}
\hline Taxon & Habitat & Voucher & TLC & ITS sequences & Publications \\
\hline Trapeliopsis granulosa (Hoffm.) Lumbsch & cor & M & - & - & Sipman \& Aptroot (2019) \\
\hline $\begin{array}{l}\text { Trapeliopsis wallrothii (Flörke ex Spreng.) } \\
\text { Hertel \& Gotth. Schneid. }\end{array}$ & ter & $\mathrm{B}, \mathrm{M}$ & - & - & Sipman \& Aptroot (2019) \\
\hline Usnea rubicunda Stirt. & cor & B & yes & - & Sipman \& Aptroot (2019) \\
\hline Usnea subscabrosa Nyl. ex Motyka & cor & $\mathrm{B}, \mathrm{M}$ & yes & - & Sipman \& Aptroot (2019) \\
\hline $\begin{array}{l}\text { Varicellaria velata (Turner) I. Schmitt } \\
\quad \text { \& Lumbsch }\end{array}$ & sax, cor & B & yes & - & Sipman \& Aptroot (2019) \\
\hline $\begin{array}{l}\text { Variospora flavescens (Huds.) Arup, Frödén } \\
\quad \text { \& Søchting }\end{array}$ & $\operatorname{sax}$ & $\mathrm{B}, \mathrm{BR}, \mathrm{M}$ & - & MN587035 & Sipman \& Aptroot (2019) \\
\hline Verrucaria macrostoma Dufour ex DC. & sax & M & - & - & Sipman \& Aptroot (2019) \\
\hline Verrucaria muralis Ach. & sax & M & - & - & Sipman \& Aptroot (2019) \\
\hline Verrucaria murina Leight. & sax & M & - & - & Sipman \& Aptroot (2019) \\
\hline Verrucaria nigrescens Pers. & sax & $\mathrm{B}, \mathrm{M}$ & - & - & Sipman \& Aptroot (2019) \\
\hline $\begin{array}{l}\text { Xanthoparmelia pulloides (Essl.) O. Blanco, } \\
\text { A. Crespo, Elix, D. Hawksw. \& Lumbsch }\end{array}$ & sax & B, M & yes & - & Sipman \& Aptroot (2019) \\
\hline $\begin{array}{l}\text { Xanthoparmelia tinctina (Maheu \& A. Gillet) } \\
\text { Hale }\end{array}$ & sax & M & - & - & $\begin{array}{l}\text { Carvalho et al. (2008) as } \\
X . \text { conspersa }\end{array}$ \\
\hline $\begin{array}{l}\text { Xanthoparmelia verruculifera (Nyl.) O. Blanco, } \\
\text { A. Crespo, Elix, D. Hawksw. \& Lumbsch }\end{array}$ & sax & B, M & yes & - & Sipman \& Aptroot (2019) \\
\hline
\end{tabular}

recognition of the shade forms relies on the black pycnidium ostioles and thick septa. The tropical species C. leptozona may be closer to I. serusiauxii because it shares the black pycnidia, but that species is saxicolous, its discs turn black, and its spores have shorter septa about half as thick as spore length. Unfortunately, no ITS sequence of this species was available in Genbank.

The only other Ikaeria species, I. aurantiellina, shares the black pycnidia and thick spore septa (spores 12-14 $\times 7-8 \mu \mathrm{m}$, septa $\sim 6-8 \mu \mathrm{m}$ thick, ratio of septum width/ spore length $0.5-0.67)$, and can be distinguished easily by the 'biatorine' apothecia (Giralt et al. 1992). However, a closer look shows that the apothecia of the two species are anatomically indistinguishable except for the somewhat shorter ascospores in I. aurantiellina. Externally there is a difference in apothecium margin color. In I. aurantiellina the margin is deep yellow to orange, slightly paler at the disc, reflecting the constant presence of anthraquinones. This gives it a biatorine appearance, but anatomically the margin contains numerous algae. In I. serusiauxii the margin is greenish grey, with more or less black pigment, and it lacks anthraquinones. This gives the apothecia a lecanorine appearance, especially when the black pigment is scarce.

The synonymy of I. aurantiellina (as Caloplaca aurantiellina) with $C$. aegatica was first suggested by Boom \& Etayo (2006), who admitted that the original description of $C$. aurantiellina is fairly different. Apparently they did not study any type material, so the synonymy may need revision.

Kondratyuk et al. (2017) mentioned two genera closely related to Ikaeria or having a similar basal root: Yoshimuria and Fominiella. The first genus is included in Figure 1, where it shows up in the Caloplacoideae. Thus it seems unrelated to our new Ikaeria species. The second contains two species: F. skii and F. tenerifensis. The ITS sequence of $F$. skii shows an affinity with the genus Athallia, as Kondratyuk et al. (2017) admit. In our
Figure 1 the species is positioned accordingly and shows no close relation with Ikaeria. For the second species, $F$. tenerifensis, no ITS sequence is available. The description and illustration of $F$. tenerifensis suggest that it differs from I. serusiauxii by the absence of black pigment in the prothallus, apothecium margins and pycnidium ostioles, and by the shorter ascospore septa, about half of spore length. The illustration presented in Kondratyuk et al. (2018, p. 179, Fig. 20) also suggests a different species.

Specimens examined (Ikaeria serusiauxii). PORTUGAL. Madeira Islands, Porto Santo: E part, SW side of Pico Juliana, saddle with Pico do Facho; $350 \mathrm{~m} ; 33^{\circ} 5.3^{\prime} \mathrm{N}, 16^{\circ} 19.4^{\prime} \mathrm{W}$; Pinus and Cupressus plantations on abandoned fields with stone walls (113). On twigs, with Cliostomum griffithii, Rinodina pruinella, Ikaeria aurantiellina; 2 March 2016; H. Sipman 62957 (B 60 0200914). id., E part, Pico do Castelo, summit area; $400 \mathrm{~m} ; 33^{\circ} 4.8^{\prime} \mathrm{N}, 16^{\circ} 20.0^{\prime} \mathrm{W}$; on Cupressus on $\mathrm{S}$ side towards parking place; 28 Feb. 2016; H. Sipman 62798 (MADJ). ITS: MN586958; LSU: MN586913; SSU: MN586907; id., E part, Pico do Castelo, summit area; $400 \mathrm{~m} ; 33^{\circ} 4.8^{\prime} \mathrm{N}$, $16^{\circ} 20.0^{\prime} \mathrm{W}$; epiphytic on S-slope; 28 Feb. 2016; H. Sipman 62802 (B 60 0200759). ITS: MN586959; LSU: MN586914; SSU: MN586908; id., E part, SW side of Pico Juliana, saddle

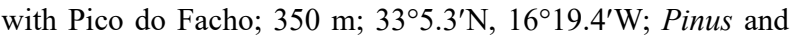
Cupressus plantations on abandoned fields with stone walls (113), on twigs, with Cliostomum griffithii, Rinodina pruinella; 2 March 2016; mixed in H. Sipman 62957 (B 60 0200914). LSU: MN586915; SSU: MN586909. Madeira: along road near Portela; $575 \mathrm{~m} ; 32^{\circ} 44.8^{\prime} \mathrm{N}, 16^{\circ} 49.6^{\prime} \mathrm{W}$; on Cedrus tree; $15 \mathrm{Apr}$. 2001; F. Schumm 13606 (B 60 0171731). id., Südöstlich von Camacha, Richtung Assomada; 500 m; epiphytisch an Malus sp., trocken-warmer Standort; 5 Oct. 1993, Kirschbaum 3075 (herb. Kirschbaum). Algarve: W of Lagos, road Vale de Boi to Barão de San Miquel, $25 \mathrm{~m} ; 37^{\circ} 05.9^{\prime} \mathrm{N}, 8^{\circ} 48.0^{\prime} \mathrm{W}$; on Ficus in orchard with Ficus and Prunus dulcis; 21 July 1993, P. van den Boom 14565 (herb. van den Boom). id., 14 km WSW of Lagos, along road to Salema, $50 \mathrm{~m} ; 37^{\circ} 04.4^{\prime} \mathrm{N}, 08^{\circ} 49.5^{\prime} \mathrm{W}$; On Ceratonia on SE slope with Ficus, Prunus dulcis and Ceratonia; 23 July 1993, P. van den Boom 14674 (herb. van den Boom), Estremadura: $25 \mathrm{~km} \mathrm{~W}$ of Setubal, area of Aldeia do Meco, $50 \mathrm{~m}$; on Ficus carica in meadow near camping; 12 Aug. 1987, P. van den Boom 
6607 (herb. van den Boom). SPAIN. Canary Islands, Fuerteventura: $7.5 \mathrm{~km} \mathrm{SSW}$ of Pájara, SW of Fayagua, Degollada del Viento, near viewpoint, $420 \mathrm{~m} ; 28^{\circ} 17.4^{\prime} \mathrm{N}, 14^{\circ} 09.2^{\prime} \mathrm{W}$; on Launaea on N slope with volcanic outcrops and shrubs; 3 March 2001, P. \& B. van den Boom 26124 (herb. van den Boom). El Hierro: W of Sabinosa, along HI-500 road, W of Montaña del Escobar, $260 \mathrm{~m} ; 27^{\circ} 45.20^{\prime} \mathrm{N}, 018^{\circ} 08.50^{\prime} \mathrm{W}$; on Juniperus on W slope, on field with volcanic outcrops, shrubs and some dead old Juniperus turbinata ssp. canariensis trees; 27 March 2009, P. \& B. van den Boom 42177 (herb. van den Boom).

Specimens examined (Ikaeria aurantiellina). PORTUGAL. Madeira Islands, Porto Santo: E part, SW side of Pico Juliana,

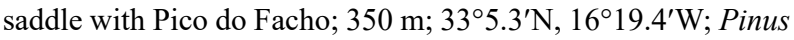
and Cupressus plantations on abandoned fields with stone walls (113), on twigs, with Cliostomum griffithii, Rinodina pruinella, Ikaeria serusiauxii; 2 March 2016; mixed in H. Sipman 62957 (B 60 0200914). ITS: MN586956; LSU: MN586911; SSU: MN586905. id., E part, lower slopes N of Pico do Facho; $\sim 350 \mathrm{~m} ; 33^{\circ} 05.2^{\prime} \mathrm{N}, 16^{\circ} 19.3^{\prime} \mathrm{W}$ : epiphytes on fallen Pinus trees on slope; 2 March 2016; H. Sipman 62969a (B 60 0200983). ITS: MN586957; LSU: MN586912; SSU: MN586906.

\section{Acknowledgements}

The participants in the fieldwork on Porto Santo, Laurens B. Sparrius, Israel Pérez-Vargas, Paula Matos, Alice Gerlach and Maaike Vervoort, are gratefully acknowledged for pleasant and effective cooperation. Sergio Perez-Ortega (Madrid, Spain) kindly arranged the permits. Access to the study area and permission to collect lichen specimens was granted by Parque Natural da Madeira (permit no. 1/PNM/2016). Pieter van den Boom (Son, The Netherlands) kindly provided additional specimens from his valuable herbarium and gave useful suggestions about identification. Arsen Gasparyan (Yerevan, Armenia) kindly helped with the DNA studies. Jan Vondrak (Průhonice, Czech Republic) gave valuable suggestions for improvement of the manuscript.

\section{References}

Arup, U., Søchting, U. \& Frödén, P. 2013. A new taxonomy of the family Teloschistaceae. Nordic Journal of Botany 31: 016-083.

Carvalho, P., Figueira, R. \& Jones, M. P. 2008. Os líquenes e fungos liquenícolas (Fungi) dos arquipélagos da Madeira e Selvagens In: Borges, P. A. V., Abreu, C., Aguiar, A. M. F., Carvalho, P., Fontinha, S., Jardim, R., Melo, I., Oliveira, P., Sequeira, M. M., Sérgio, C., Serrano, A. R. M., Sim-Sim, M. \& Vieira, P., Listagem dos fungos, flora e fauna terrestres dos arquipélagos da Madeira e Selvagens. p. 95-103, [105]-122. Funchal, Angra do Heroísmo: Direcção Regional do Ambiente da Madeira and Universidade dos Açores.

Fletcher, A. \& Laundon, J. R. 2009. Caloplaca. In: Smith, C. W., Aptroot, A., Coppins, B. J., Fletcher, A., Gilbert, O. L., James, P. W. $\&$ Wolseley, P. A. (eds), The lichens of Great Britain and Ireland, pp. 245-273. The British Lichen Society, Department of Botany, The Natural History Museum, London.

Follmann, G. 1990. Zur Kenntnis der Flechtenflora und Flechtenvegetation von Madeira und den umliegenden Inseln. I. Chorologischsoziologischer Abriss. Courier Forschungsinstitut Senckenberg 129: 91-102.

Giralt, M., Nimis, P. L. \& Poelt, J. 1992. Studien über den Formenkreis von Caloplaca flavorubescens in Europa. Cryptogamie, BryologieLichénologie 13: 261-273.
Haugan, R. 1992. Anzia centrifuga, a new lichen species from Porto Santo, Madeira. Mycotaxon 44: 45-50.

Hoang, D. T., Chernomor, O., Haeseler, A. von, Minh, B. Q. \& Vinh, L. S. 2018. UFBoot2: Improving the ultrafast bootstrap approximation. Molecular Biology and Evolution 35: 518-522.

Kondratyuk, S. Y., Lokös, L., Upreti, D. K., Nayaka, S., Mishra, G. K., Ravera, S., Jeong, M.-H., Jang, S.-H., Park, J. S. \& Hur, J.-S. 2017. New monophyletic branches of the Teloschistaceae (lichen-forming ascomycota) proved by three gene phylogeny. Acta Botanica Hungarica 59: 71-136.

Kondratyuk, S. Y., Lokös, L., Halda, J. P., Farkas, E., Upreti, D. K., Thell, A., Woo, J.-J., Oh, S.-O. \& Hur, J.-S. 2018. New and noteworthy lichen-forming and lichenicolous Fungi 7. Acta Botanica Hungarica 60: 115-184.

Krog, H. 1990. New Ramalina species from Porto Santo, Madeira. The Lichenologist 22: 241-247.

Krog, H. \& Østhagen, H. 1980. Two new Ramalina species from Porto Santo, the Madeira Islands. Norwegian Journal of Botany 27: $185-188$.

Kukwa, M., Schmitt, I. \& Ertz, D. 2018. Ochrolechia incarnata comb. nov. (Lecanoromycetes, Ascomycota), a distinct species of the O. parella group from Europe and Macaronesia. - Phytotaxa 371(2): 119-126.

Meyer, B. 2002. Die Flechtengattung Clauzadea. Sendtnera 8: 85-154.

Nguyen, L.-T., Schmidt, H. A., Haeseler, A von, \& B. Q. Minh, B. Q. 2015. IQ-TREE: A fast and effective stochastic algorithm for estimating maximum likelihood phylogenies. Molecular Biology and Evolution 32: 268-274.

Pitard, J. \& Harmand, J. 1911. Contribution à l'étude des lichens des îles Canaries. Bulletin de la Societé Botanique de France 58, Mém. 22: $1-72$.

Sérusiaux, E., van den Boom, P. \& Ertz, D. 2010. A two-gene phylogeny shows the lichen genus Niebla (Lecanorales) is endemic to the New World and does not occur in Macaronesia nor in the Mediterranean basin. Fungal Biology 114: 528-537.

Sipman, H. \& Aptroot, A. 2019. Pictures of PORTO SANTO lichens and lichenicolous fungi. https://archive.bgbm.org/sipman/Zschackia/ PortoSanto/genuslist.htm [14 Aug. 2019]

Šoun, J., Vondrak, J., Søchting, U., Hrouzek, P., Khodosovtsev, A. \& Arup, U. 2011. Taxonomy and phylogeny of the Caloplaca cerina group in Europe. The Lichenologist 43: 113-135.

Sparrius, L. B., Aptroot, A., Sipman, H. J. M., Pérez-Vargas, I., Matos, P., Gerlach, A. \& Vervoort, M. 2017. Estimating the population size of the endemic lichens Anzia centrifuga (Parmeliaceae) and Ramalina species (Ramalinaceae) on Porto Santo (Madeira Archipelago). The Bryologist 120: 293-301.

Tehler, A., Dahlkild, A., Eldenas, P. \& Feige, G. B. 2004. The phylogeny and taxonomy of Macaronesian, European and Mediterranean Roccella (Roccellaceae, Arthoniales). Symbolae Botanicae Upsalienses 34: 405-428.

Tehler, A., Ertz, D. \& Irestedt, M. 2013. The genus Dirina (Roccellaceae, Arthoniales) revisited. - The Lichenologist 45(4): 427-476.

Timdal, E. 1992. A monograph of the genus Toninia (Lecideaceae, Ascomycetes). Opera Botanica 110: 1-137.

Trifinopoulos, J., Nguyen, L.-T., Haeseler, A. von \& Minh, B. Q. 2016. W-IQ-TREE: a fast online phylogenetic tool for maximum likelihood analysis. Nucleic Acids Research 44 (W1): W232-W235.

Van den Boom, P. P. G. \& Etayo, J. 2006. New records of lichens and lichenicolous fungi from Fuerteventura (Canary Islands), with descriptions of some new species. Cryptogamie, Mycology 27: 341-374.

Zakeri, Z., Divakar, P. K. \& Otte, V. 2017. Taxonomy and phylogeny of Aspiciliella, a resurrected genus of Megasporaceae, including the new species A. portosantana. Herzogia 30: 166-176. 